\title{
Replica symmetry breaking in attractor neural network models
}

\author{
Helmut Steffan* and Reimer Kühn ${ }^{\dagger}$ \\ Institut für Theoretische Physik \\ Ruprecht-Karls-Universität Heidelberg \\ Philosophenweg 19 \\ 69120 Heidelberg, Germany
}

Submitted to Zeitschrift für Physik B

\begin{abstract}
The phenomenon of replica symmetry breaking is investigated for the retrieval phases of Hopfield-type network models. The basic calculation is done for the generalized version of the standard model introduced by Horner [1] and by Perez-Vicente and Amit [2] which can exhibit low mean levels of neural activity. For a mean activity $\bar{a}=1 / 2$ the Hopfield model is recovered. In this case, surprisingly enough, we cannot confirm the well known one step replica symmetry breaking (1RSB) result for the storage capacity which was presented by Crisanti, Amit and Gutfreund [3] $\left(\alpha_{c}^{1 \mathrm{RSB}} \simeq 0.144\right)$. Rather, we find that 1RSB- and 2RSB-Ansätze yield only slightly increased capacities as compared to the replica symmetric value $\left(\alpha_{c}^{1 \mathrm{RSB}} \simeq 0.138186\right.$ and $\alpha_{c}^{2 \mathrm{RSB}} \simeq 0.138187$ compared to $\left.\alpha_{c}^{\mathrm{RS}} \simeq 0.137905\right)$, significantly smaller also than the value $\alpha_{c}^{\text {sim }}=0.145 \pm 0.009$ reported from simulation studies. These values still lie within the recently discovered reentrant phase [4]. We conjecture that in the infinite Parisi-scheme the reentrant behaviour disappears as is the case in the SK-spin-glass model (Parisi-Toulouse-hypothesis). The same qualitative results are obtained in the low activity range.
\end{abstract}

*supported by Sonderforschungsbereich 123 'Stochastische Mathematische Modelle'

${ }^{\dagger}$ supported by a Heisenberg fellowship 
H. Steffan and R. Kühn:

\section{Introduction}

The Hopfield model of a neural network [5] is at present considered to be well understood. By suitably adopting mean field theory of spin glass like systems [6], Amit et al. [7] were able to compute its phase diagram in a replica symmetric $(\mathrm{RS})$ approximation. In particular, they found the $T=0$ storage capacity of the model to be $\alpha_{c}^{\mathrm{RS}} \simeq 0.138$ in the RS framework. From simulations, they obtained a slightly larger value $\alpha_{c}^{\text {sim }}=0.145 \pm 0.009$ [7], and they conjectured that the origin of such a discrepancy might be put down to effects of replica symmetry breaking (RSB).

A subsequent one-step replica symmetry breaking (1RSB) analysis of Crisanti et al. [3] did indeed yield $\alpha_{c}^{1 \mathrm{RSB}} \simeq 0.144$ in very good agreement with the earlier simulation results, so that the question of the storage capacity of this model appeared to have been settled: effects of RSB are found to be small and 1RSB corrections seem to bridge the gap between RS results and those of numerical simulations. Large scale simulations by Kohring [8] seemed to confirm the overall picture.

For generalized Hopfield-type models adapted to store an ensemble of low-activity patterns $[1,2]$, deviations between results of RS mean field analysis and simulation data were found to be much stronger. For instance, at an intermediate level $\bar{a}=0.1$ for the mean activity, considered to be in a neurophysiologically acceptable range, it was found that $\alpha_{c}^{\mathrm{RS}} \simeq 0.483$ [1], whereas simulations [9] yielded $\alpha_{c}^{\mathrm{sim}}=0.585 \pm 0.01$, i.e. a discrepancy of roughly $20 \%$ as opposed to a $4 \%$ discrepancy in the standard model.

The question then arises, whether in such a situation, a 1RSB analysis would likewise be sufficient to close the gap between RS results and estimates obtained from Monte-Carlo simulations, or whether - on contrary - further steps in Parisi's approximating scheme [10] or even the full hierarchical scheme of RSB [11] were needed to explain the numerical results.

In the present paper, we have addressed this question, and we have obtained answers in completly unanticipated directions, as follows. We have performed 1RSB and 2RSB analyses for general Hopfield-type models storing ensembles of low-activity patterns [1], [2]. For an activity $\bar{a}=1 / 2$, these models are equivalent to Hopfield's standard model [5], and our 1RSB results should therefore merge with those of Crisanti et al. [3] as we take the limit $\bar{a} \rightarrow 1 / 2$. It turns out that such 'confirmation en route' of the findings of Crisanti et al. could not be accomplished, and we are forced to conclude that their often quoted value $\alpha_{c}^{1 \mathrm{RSB}} \simeq 0.144$ for the storage capacity of the standard model is in error - in particular since we have invited an independent check of our results, which was recently performed by Huyghebaert [12] using the bifurcation-finding software package 'AUTO'.

Our main results for the $\bar{a}=1 / 2$ case are $\alpha_{c}^{1 \mathrm{RSB}} \simeq 0.138186$, and $\alpha_{c}^{2 \mathrm{RSB}} \simeq 0.138187$. These values are still lying well within the recently discovered reentrance phase of the Hopfield model [4]. That is, 1RSB and 2RSB approximations are found insufficient to bridge the gap between RS and simulation results. Moreover, we shall argue on the basis of the Parisi-Toulouse hypothesis [13] appropriatly adapted to the Hopfield model phase diagram that even the full hierarchical RSB scheme of Parisi [11] will not yield storage capacities as high as those reported from various simulation studies. Rather, as in the 
SK-model, the reentrance phenomenon is expected to simply disappear in the full RSB solution.

This renders the problem of how to explain discrepancies between theory and numerical experiment an open question again.

For values other than $\bar{a}=1 / 2$ we obtain qualitatively similar results. Here, we have performed numerical evaluations only of the 1RSB theory, and we find the 1RSB storage capacity to lie again within the RS reentrance phase (as it should according to the ParisiToulouse hypothesis), $\alpha_{c}^{1 \mathrm{RSB}} \simeq 0.495$ at $\bar{a}=0.1$, well below the simulation result $\alpha_{c}^{\text {sim }}=$ $0.585 \pm 0.01[9]$.

Our paper is organized as follows. In Sec. 2, we introduce the generalized Hopfield model for the storage of low-activity patterns. In Sec. 3, we compute the replica free energy and evaluate it in the RS, and the 1RSB and 2RSB approximations, relegating details of the derivations to appendices. In Sec. 4 we present and discuss outcomes of a numerical analysis of the $1 \mathrm{RSB}$ and the $2 \mathrm{RSB}$ approximations for the Hopfield model, and of the 1RSB approximation for the generalized model at $\bar{a}=0.1$. In Sec. 5, we discuss analogies between the Hopfield model and the SK-model with ferromagnetic anisotropy [6] to put our results into a wider perspective. From an appropriate adaption of the Parisi-Toulouse hypothesis to the phase diagrams of the generalized Hopfield-type models, we conjecture that even the full hierarchical RSB solution of these models, presumably providing their exact solution, would yield results which are at variance with currently available simulation data. A concluding section is devoted to discuss the state of affairs that has thus emerged.

\section{The Generalized Hopfield Model}

In order to set the scene and to fix our notation, we shall here introduce our variant of the generalized Hopfield model for the storage of patterns of arbitrary activity.

Let us denote by $\bar{a}$ the average fraction of active nodes in each pattern to be stored. We take a storage prescription of a generalized Hebbian form

$$
J_{i j}=\frac{1}{N} \sum_{\mu=1}^{p} \xi_{i}^{\mu} \xi_{j}^{\mu}, \quad i \neq j,
$$

where $1 \leq i, j \leq N$ label the neurons of the net and $1 \leq \mu \leq p$ enumerates the patterns. For the representation of the active $(A)$ and inactive $(I)$ neural states $s_{i}$, we take

$$
A=\sqrt{\frac{1-\bar{a}}{\bar{a}}}, \quad I=-\sqrt{\frac{\bar{a}}{1-\bar{a}}},
$$

given that the pattern statistics is

$$
\xi_{i}^{\mu}= \begin{cases}A, & \text { with prob. } \quad \bar{a} \\ I, & \text { with prob. } 1-\bar{a}\end{cases}
$$


The representation defined by (2) is known to be well adapted to the storage of patterns with statistics given in (3) [1],[2], using a generalized Hebbian storage prescription as in (1), and supplementing it with a suitable threshold $\vartheta$. Moreover, it is known to saturate the well known Gardner bound [14]

$$
\alpha_{c} \sim \frac{1}{2 \bar{a}|\ln \bar{a}|}
$$

as $\bar{a} \rightarrow 0$. The asynchronous dynamics of the model, defined by

$$
s_{i}(t+\Delta t)=A \theta\left(h_{i}(t)-\vartheta\right)+I\left(1-\theta\left(h_{i}(t)-\vartheta\right)\right),
$$

with $h_{i}(t)=\sum_{j} J_{i j} s_{j}(t)$ and $\theta(x)$ the usual Heaviside function, is governed by the energy function

$$
H[s]=-\frac{1}{2} \sum_{i \neq j} J_{i j} s_{i} s_{j}+\vartheta \sum_{i} s_{i}
$$

Asynchronous Glauber dynamics, if adapted to (5), converges to a Gibbs distribution over the space of neural states that is generated by (6). The next section is devoted to deal with the quenched randomness in the couplings due to the stored patterns.

\section{The Replica Free Energy}

\subsection{General Theory}

As usual, to investigate the thermodynamics of systems with quenched randomness, one has to compute the quenched free energy $-\beta f(\beta)=\langle\ln Z\rangle_{\xi}$, where $Z$ is the partition function at fixed disorder and where $\langle\ldots\rangle_{\xi}$ denotes an average over disorder according to its distribution. The computation utilizes the replica identity

$$
\langle\ln Z\rangle_{\xi}=\lim _{n \rightarrow 0} \frac{1}{n} \ln \left\langle Z^{n}\right\rangle_{\xi}
$$

In the details of the calculation we follow Amit et al. [7]. Assuming that the system state has macroscopic correlations only with a finite number of patterns, say $\xi^{\nu}, \quad \nu=$ $1, \ldots, l$, we obtain the replica free energy through standard arguments [7], by averaging over the remaining patterns $\xi^{\mu}$ :

$$
n f=\frac{1}{2} \sum_{\nu, a}\left(m_{a}^{\nu}\right)^{2}+\frac{\alpha}{2 \beta} \ln \operatorname{det}(\mathbf{1}-\beta \mathbf{q})+\beta \alpha \sum_{a \leq b} r_{a b} q_{a b}-\frac{1}{\beta}\langle\ln \hat{Z}\rangle_{\xi^{\nu}}
$$

Here $\hat{Z}$ is a replica partition function

$$
\hat{Z}=\sum_{\left\{s^{a}\right\}} \exp \left\{\beta\left(\sum_{a} s^{a}\left[\sum_{\nu} m_{a}^{\nu} \xi^{\nu}-\vartheta\right]+\beta \alpha \sum_{a \leq b} r_{a b} s^{a} s^{b}-\frac{\alpha}{2} \sum_{a}\left(s^{a}\right)^{2}\right)\right\},
$$


corresponding to a replicated single-site Hamiltonian of the form

$$
\hat{H}=-\sum_{a} s^{a}\left[\sum_{\nu} m_{a}^{\nu} \xi^{\nu}-\vartheta\right]-\beta \alpha \sum_{a \leq b} r_{a b} s^{a} s^{b}+\frac{\alpha}{2} \sum_{a}\left(s^{a}\right)^{2} .
$$

We have introduced overlaps with the condensed patterns

$$
m_{a}^{\nu}=\frac{1}{N} \sum_{i} \xi_{i}^{\nu} s_{i}^{a}
$$

and the matrix $\mathbf{q}$ of Edwards-Anderson order parameters, with elements

$$
q_{a b}=\frac{1}{N} \sum_{i} s_{i}^{a} s_{i}^{b}
$$

with $1 \leq a, b \leq n$ labeling the replicas. In mean-field theory, the order parameters must satisfy the fixed point equations

$$
\begin{aligned}
m_{a}^{\nu} & =\left\langle\xi^{\nu}\left\langle s^{a}\right\rangle\right\rangle_{\xi^{\nu}}, \quad \nu=1, \ldots, l, \quad a=1, \ldots, n \\
q_{a b} & =\left\langle\left\langle s^{a} s^{b}\right\rangle\right\rangle_{\xi^{\nu}}, \quad 1 \leq a, b \leq n,
\end{aligned}
$$

in which $\langle\ldots\rangle$ without subscript denotes a Gibbs average performed with the Hamiltonian $\hat{H}$ in (10) while $\langle\ldots\rangle_{\xi^{\nu}}$ designates an average over the condensed patterns $\xi^{\nu}$. The matrix $\mathbf{r}$ with elements $r_{a b}$ is simply related to the q-matrix. One has

$$
\beta r_{a a}=\frac{1}{2}(\mathbf{1}-\beta \mathbf{q})_{a a}^{-1}, \quad \beta r_{a b}=(\mathbf{1}-\beta \mathbf{q})_{a b}^{-1}, \quad a<b,
$$

with 1 the $n \times n$ unit matrix. It is understood that an analytic continuation to non-integer $n$ and the $n \rightarrow 0$ limit are eventually to be taken.

Equations (13)-(14) are usually solved by making an ansatz concerning the transformation properties of the saddle-point values of the order parameters $m_{a}^{\nu}$ and $q_{a b}$ under permutation of replicas.

\subsection{The replica symmetric approximation}

The first and most natural ansatz is of course that exhibiting complete replica symmetry $(\mathrm{RS})$ :

$$
m_{a}^{\nu}=m^{\nu}, \quad q_{a a}=\hat{q}, \quad q_{a b}=q, \quad a \neq b,
$$

the replica symmetry of $\mathbf{q}$ being inherited by $\mathbf{r}$ due to (14). This ansatz allows for an easy evaluation of all terms appearing in (8), (13) and (14), as well as for an analytic continuation of the results to $n \rightarrow 0$. As the $n \rightarrow 0$ limit is taken one gets

$$
\begin{aligned}
f= & \frac{1}{2} \sum_{\nu}\left(m^{\nu}\right)^{2}-\frac{1}{\beta}\langle\ln \tilde{Z}\rangle_{z, \xi^{\nu}} \\
& +\frac{\alpha}{2}\left(\frac{1}{\beta} \ln (1-\beta(\hat{q}-q))-\frac{q}{1-\beta(\hat{q}-q)}+2 \beta \hat{r} \hat{q}-\beta r q\right),
\end{aligned}
$$


where $m^{\nu}, \hat{q}$ and $q$ satisfy

$$
\begin{aligned}
m^{\nu} & =\left\langle\xi^{\nu}\langle s\rangle\right\rangle_{z, \xi^{\nu}} \\
\hat{q} & =\left\langle\left\langle s^{2}\right\rangle\right\rangle_{z, \xi^{\nu}} \\
q & =\left\langle\langle s\rangle^{2}\right\rangle_{z, \xi^{\nu}}
\end{aligned}
$$

Here $\langle\ldots\rangle$ without subscript denotes a 'thermal average' performed over the Gibbs distribution generated by the single-site Hamiltonian

$$
\tilde{H}=-s\left[\sum_{\nu} m^{\nu} \xi^{\nu}-\vartheta+\sqrt{\alpha r} z\right]-s^{2} \frac{\alpha}{2}(\beta(2 \hat{r}-r)-1),
$$

while $\tilde{Z}$ is the corresponding partition function, and $\langle\ldots\rangle_{z, \xi^{\nu}}$ a combined average over a zero mean unit variance Gaussian $z$ and the $\xi^{\nu}$ according to their distribution. Moreover, we have

$$
r=\frac{q}{[1-\beta(\hat{q}-q)]^{2}} \quad \beta(2 \hat{r}-r)=\frac{1}{1-\beta(\hat{q}-q)} .
$$

The computation leading to these equations are standard, and we shall not document them here.

The RS solution fails to be thermodynamically stable as the temperature is lowered through the AT-line [15], given by

$$
T^{2}=\frac{\alpha}{(1-C)^{2}}\left\langle\left(\left\langle s^{2}\right\rangle-\langle s\rangle^{2}\right)^{2}\right\rangle_{z, \xi}
$$

where we have introduced the 'response parameter' $C=\beta(\hat{q}-q)$. The RS Hopfield model results are recovered by taking $\vartheta=0$ and $A=-I=1$ in (16)-(20).

To improve upon the results of the $\mathrm{RS}$ approximation in the region where $\mathrm{RS}$ is known to be broken according to the AT criterion [15], one can follow Parisi's scheme of hierarchical replica symmetry breaking $[10,11]$. In what follows, we present the first two steps of Parisi's approximations for the generalized Hopfield model. Details of the calculation are to be found in appendices $\mathrm{A}$ and $\mathrm{B}$ for the $1 \mathrm{RSB}$ and the $2 \mathrm{RSB}$ approximations respectively.

\subsection{The 1RSB approximation}

In the $1 \mathrm{RSB}$ ansatz, one assumes that the overlaps $m_{a}^{\nu}$ still exhibit the full invariance with respect to permutations of replicas,

$$
m_{a}^{\nu}=m^{\nu}, \quad a=1, \ldots, n,
$$

whereas the Edwards-Anderson matrix $\mathbf{q}$ acquires the following structure,

$$
q_{a b}=\left\{\begin{array}{lr}
\hat{q}, & a=b \\
q_{1}, & |a-b| \leq m \\
q_{0}, & \text { otherwise }
\end{array}\right.
$$


Here $m<n$ is a partitioning parameter that is to be determined from a stationarity condition for the free energy. Formally, we may express $\mathbf{q}$ in terms of a tensor product structure

$$
\mathbf{q}=\left(\hat{q}-q_{1}\right) \mathbf{1}_{n}+\left(q_{1}-q_{0}\right) \mathbf{1}_{\frac{n}{m}} \otimes \mathbf{e}_{m} \mathbf{e}_{m}^{\mathrm{T}}+q_{0} \mathbf{e}_{n} \mathbf{e}_{n}^{\mathrm{T}},
$$

where $\mathbf{1}_{k}$ denotes a $k$-dimensional unit matrix and $\mathbf{e}_{k}^{\mathrm{T}}=(1,1, \ldots, 1)$ a transposed column vector with $k$ elements identical 1 , that is, $\mathbf{e}_{k} \mathbf{e}_{k}^{\mathrm{T}}$ is nothing but a $k \times k$ matrix completely filled with one's. The matrix $\mathbf{1}-\beta \mathbf{q}$ clearly has the same type of tensorial structure, and so does $\mathbf{r}$, because it is simply related with its inverse. These observations allow a fairly straightforward evaluation of all terms appearing in (8)-(10), as well as of a 1RSB formulation of the saddle point equations (13). We get (for details, see appendix A)

$$
\begin{aligned}
& f\left(m^{\nu}, q_{0}, q_{1}, \hat{q}, r_{0}, r_{1}, \hat{r} ; m\right)=\frac{1}{2} \sum_{\nu}\left(m^{\nu}\right)^{2}-\frac{1}{\beta m}\left\langle\ln \left\langle\tilde{Z}^{m}\right\rangle_{z_{1}}\right\rangle_{z, \xi^{\nu}} \\
& +\frac{\alpha}{2}\left(-\frac{q_{0}}{Q_{q_{0}}}+\frac{1}{\beta m} \ln \left(\frac{Q_{q_{0}}}{Q_{q_{1}}}\right)+\frac{1}{\beta} \ln Q_{q_{1}}+\beta 2 \hat{r} \hat{q}+\beta r_{1} q_{1}(m-1)-\beta r_{0} q_{0} m\right)
\end{aligned}
$$

for the free energy, and

$$
\begin{aligned}
& m^{\nu}=\left\langle\xi^{\nu} \frac{\left\langle\tilde{Z}^{m}\langle s\rangle\right\rangle_{z_{1}}}{\left\langle\tilde{Z}^{m}\right\rangle_{z_{1}}}\right\rangle_{z, \xi^{\nu}} \\
& q_{0}=\left\langle\left(\frac{\left\langle\tilde{Z}^{m}\langle s\rangle\right\rangle_{z_{1}}}{\left\langle\tilde{Z}^{m}\right\rangle_{z_{1}}}\right)^{2}\right\rangle_{z, \xi^{\nu}} \\
& q_{1}=\left\langle\frac{\left\langle\tilde{Z}^{m}\langle s\rangle^{2}\right\rangle_{z_{1}}}{\left\langle\tilde{Z}^{m}\right\rangle_{z_{1}}}\right\rangle_{z, \xi^{\nu}} \\
& C \equiv \beta\left(\hat{q}-q_{1}\right)=\frac{1}{\sqrt{\alpha \Delta r_{1}}}\left\langle\frac{\left\langle\tilde{Z}^{m} \frac{d}{d z_{1}}\langle s\rangle\right\rangle_{z_{1}}}{\left\langle\tilde{Z}^{m}\right\rangle_{z_{1}}}\right\rangle_{z, \xi^{\nu}}
\end{aligned}
$$

for the fixed point equations. There is an extra equation due to the stationary condition on $f$ with respect to the partitioning parameter $m$, which can be expressed as follows:

$$
\begin{aligned}
-\frac{1}{\beta m}\left\langle\ln \left\langle\tilde{Z}^{m}\right\rangle_{z_{1}}\right\rangle_{z, \xi^{\nu}} & =-\frac{1}{\beta m}\left\langle\frac{\left\langle\tilde{Z}^{m} \ln \tilde{Z}^{m}\right\rangle_{z_{1}}}{\left\langle\tilde{Z}^{m}\right\rangle_{z_{1}}}\right\rangle_{z, \xi^{\nu}} \\
+ & \frac{\alpha}{2}\left(\frac{q_{0}}{Q_{q_{0}}}-\frac{q_{1}}{Q_{q_{1}}}-\frac{1}{\beta m} \ln \left(\frac{Q_{q_{0}}}{Q_{q_{1}}}\right)\right)
\end{aligned}
$$

In (24 - 26), we have introduced the auxiliary quantities $Q_{q_{i}}$ defined as

$$
Q_{q_{1}}=1-\beta\left(\hat{q}-q_{1}\right) \quad, \quad Q_{q_{0}}=Q_{q_{1}}-\beta m\left(q_{1}-q_{0}\right) .
$$


The elements of the r-matrix, are given by

$$
\begin{aligned}
& r_{0} \equiv \Delta r_{0}=\frac{\Delta q_{0}}{Q_{q_{0}}^{2}}, \\
& r_{1}-r_{0} \equiv \quad \Delta r_{1}=\frac{\Delta q_{1}}{Q_{q_{0}} Q_{q_{1}}}, \\
& \beta\left(2 \hat{r}-r_{1}\right) \equiv \quad \beta \Delta \hat{r}=\frac{1}{Q_{q_{1}}},
\end{aligned}
$$

where $\Delta q_{0}=q_{0}$ and $\Delta q_{1}=q_{1}-q_{0}$. The quantity $\tilde{Z}$, finally, denotes the partition function corresponding to the single-site Hamiltonian

$$
\tilde{H}=-\left[\sum_{\nu} m^{\nu} \xi^{\nu}+\sqrt{\alpha \Delta r_{0}} z+\sqrt{\alpha \Delta r_{1}} z_{1}-\vartheta\right] s-\frac{\alpha}{2}[\beta \Delta \hat{r}-1] s^{2}
$$

The notation used for the averaging brackets conforms to that introduced in the previous subsection.

The numerical solution of these equations will be discussed in Sect. 4 below. The case of the standard Hopfield model is recovered by taking $A=-I=1$ and $\vartheta=0$.

\subsection{The 2RSB approximation}

The 2RSB approximation is obtained from the 1RSB scheme by endowing the $m \times m$ diagonal submatrices of $\mathbf{q}$ with a structure akin to that arrived at when breaking $\mathrm{RS}$ for the first time in the full matrix. Formally,

$$
\mathbf{q}=\left(\hat{q}-q_{2}\right) \mathbf{1}_{n}+\left(q_{2}-q_{1}\right) \mathbf{1}_{\frac{n}{m_{2}}} \otimes \mathbf{e}_{m_{2}} \mathbf{e}_{m_{2}}^{\mathrm{T}}+\left(q_{1}-q_{0}\right) \mathbf{1}_{\frac{n}{m_{1}}} \otimes \mathbf{e}_{m_{1}} \mathbf{e}_{m_{1}}^{\mathrm{T}}+q_{0} \mathbf{e}_{n} \mathbf{e}_{n}^{\mathrm{T}}
$$

The tensorial structure of $\mathbf{q}$ is inherited by $\mathbf{1}-\beta \mathbf{q}$ and by $\mathbf{r}$ for the same reasons as in the $1 \mathrm{RSB}$ case. Moreover, as in the $1 \mathrm{RSB}$ approximation, one keeps replica symmetry for the overlaps $m_{a}^{\nu}$. This leads to the free energy

$$
\begin{aligned}
f\left(m^{\nu}, q_{0}, q_{1},\right. & \left.q_{2}, \hat{q}, r_{0}, r_{1}, r_{2}, \hat{r} ; m_{1}, m_{2}\right)=\frac{1}{2} \sum_{\nu}\left(m^{\nu}\right)^{2}-\frac{1}{\beta m_{1}}\left\langle\ln \left\langle\left\langle\tilde{Z}^{m_{2}}\right\rangle_{z_{2}}^{\frac{m_{1}}{m_{2}}}\right\rangle_{z_{1}}\right\rangle_{z, \xi^{\nu}} \\
+ & \frac{\alpha}{2}\left(-\frac{q_{0}}{Q_{q_{0}}}+\frac{1}{\beta m_{1}} \ln \left(\frac{Q_{q_{0}}}{Q_{q_{1}}}\right)+\frac{1}{\beta m_{2}} \ln \left(\frac{Q_{q_{1}}}{Q_{q_{2}}}\right)+\frac{1}{\beta} \ln Q_{q_{2}}\right. \\
+ & \left.\beta 2 \hat{r} \hat{q}+\beta r_{2} q_{2}\left(m_{2}-1\right)+\beta r_{1} q_{1}\left(m_{1}-m_{2}\right)-\beta r_{0} q_{0} m_{1}\right)
\end{aligned}
$$

and the saddle point equations

$$
m^{\nu}=\left\langle\xi^{\nu} \frac{\left\langle\left\langle\tilde{Z}^{m_{2}}\right\rangle_{z_{2}}^{\frac{m_{1}}{m_{2}}} \frac{\left\langle\tilde{Z}^{m_{2}\langle s\rangle}\right\rangle_{z_{2}}}{\left\langle\tilde{Z}^{m_{2}}\right\rangle_{z_{2}}}\right\rangle_{z_{1}}}{\left\langle\left\langle\tilde{Z}^{m_{2}}\right\rangle_{z_{2}}^{\frac{m_{1}}{m_{2}}}\right\rangle_{z_{1}}}\right\rangle_{z, \xi^{\nu}}
$$


Replica symmetry breaking in attractor neural network models

$$
\begin{aligned}
& q_{0}=\left\langle\left(\frac{\left\langle\left\langle\tilde{Z}^{m_{2}}\right\rangle_{z_{2}}^{\frac{m_{1}}{m_{2}}} \frac{\left\langle\tilde{Z}^{m_{2}}\langle s\rangle\right\rangle_{z_{2}}}{\left\langle\tilde{Z}^{m_{2}}\right\rangle_{z_{2}}}\right\rangle_{z_{1}}}{\left\langle\left\langle\tilde{Z}^{m_{2}}\right\rangle_{z_{2}}^{\frac{m_{1}}{m_{2}}}\right\rangle_{z_{1}}}\right\rangle_{z, \xi^{\nu}}^{2}\right.
\end{aligned}
$$

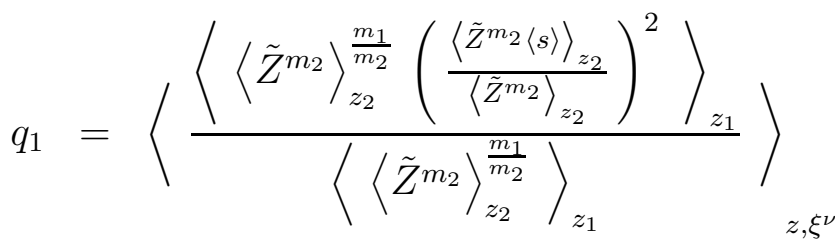

$$
\begin{aligned}
& q_{2}=\left\langle\frac{\left\langle\left\langle\tilde{Z}^{m_{2}}\right\rangle_{z_{2}}^{\frac{m_{1}}{m_{2}}} \frac{\left\langle\tilde{Z}^{m_{2}}\langle s\rangle^{2}\right\rangle_{z_{2}}}{\left\langle\tilde{Z}^{m_{2}}\right\rangle_{z_{2}}}\right\rangle_{z_{1}}}{\left\langle\left\langle\tilde{Z}^{m_{2}}\right\rangle_{z_{2}}^{\frac{m_{1}}{m_{2}}}\right\rangle_{z_{1}}}\right\rangle
\end{aligned}
$$

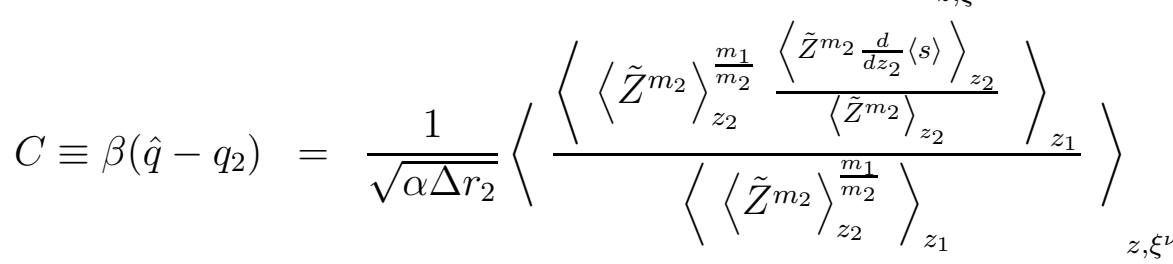

The partitioning parameters $m_{1}$ and $m_{2}$ are determined by stationarity conditions on $f$, leading to two further fixed point equations, namely:

$$
\begin{aligned}
& -\frac{1}{\beta m_{1}}\left\langle\ln \left\langle\left\langle\tilde{Z}^{m_{2}}\right\rangle_{z_{2}}^{\frac{m_{1}}{m_{2}}}\right\rangle_{z_{1}}\right\rangle_{z, \xi^{\nu}}=-\frac{1}{\beta m_{2}}\left\langle\frac{\left\langle\left\langle\tilde{Z}^{m_{2}}\right\rangle_{z_{2}}^{\frac{m_{1}}{m_{2}}} \ln \left\langle\tilde{Z}^{m_{2}}\right\rangle_{z_{2}}\right\rangle_{z_{1}}}{\left\langle\left\langle\tilde{Z}^{m_{2}}\right\rangle_{z_{2}}^{\frac{m_{1}}{m_{2}}}\right\rangle_{z_{1}}}\right\rangle_{z, \xi^{\nu}} \\
& +\frac{\alpha}{2}\left(\frac{q_{0}}{Q_{q_{0}}}-\frac{q_{1}}{Q_{q_{1}}}-\frac{1}{\beta m_{1}} \ln \left(\frac{Q_{q_{0}}}{Q_{q_{1}}}\right)\right)
\end{aligned}
$$

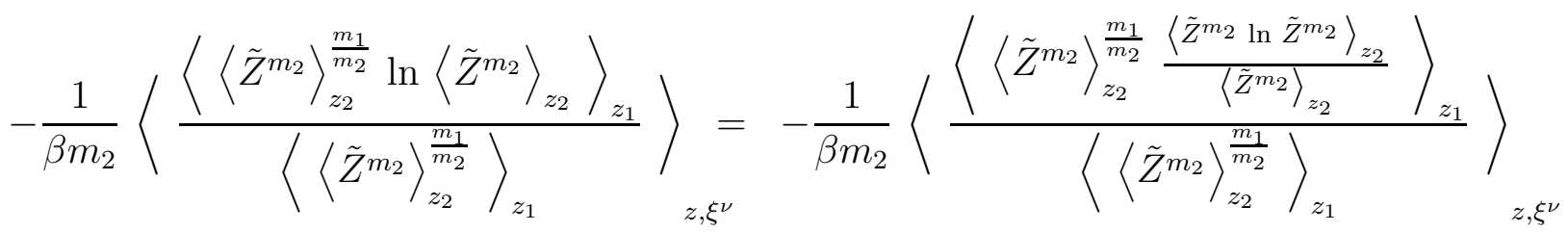

$$
\begin{aligned}
& +\frac{\alpha}{2}\left(\frac{q_{1}}{Q_{q_{1}}}-\frac{q_{2}}{Q_{q_{2}}}-\frac{1}{\beta m_{2}} \ln \left(\frac{Q_{q_{1}}}{Q_{q_{2}}}\right)\right)
\end{aligned}
$$

The $\mathbf{r}$ elements, finally, are algebraically related to the elements of $\mathbf{q}$ :

$$
r_{0} \equiv \Delta r_{0}=\frac{\Delta q_{0}}{Q_{q_{0}}^{2}}
$$




$$
\begin{aligned}
& r_{1}-r_{0} \equiv \Delta r_{1}=\frac{\Delta q_{1}}{Q_{q_{0}} Q_{q_{1}}} \\
& r_{2}-r_{1} \equiv \quad \Delta r_{2}=\frac{\Delta q_{2}}{Q_{q_{1}} Q_{q_{2}}}, \\
& \beta\left(2 \hat{r}-r_{2}\right) \equiv \quad \beta \Delta \hat{r}=\frac{1}{Q_{q_{2}}} .
\end{aligned}
$$

Here $\Delta q_{0}=q_{0}$, and $\Delta q_{i}=q_{i}-q_{i-1}$ for $i=1,2$, while

$$
Q_{q_{2}}=1-\beta\left(\hat{q}-q_{2}\right), \quad Q_{q_{1}}=Q_{q_{2}}-\beta m_{2}\left(q_{2}-q_{1}\right), \quad Q_{q_{0}}=Q_{q_{1}}-\beta m_{1}\left(q_{1}-q_{0}\right) .
$$

As in the previous subsection, $\tilde{Z}$ denotes a partition function corresponding to a single-site Hamiltonian, namely

$$
\tilde{H}=-\left[\sum_{\nu} m^{\nu} \xi^{\nu}+\sqrt{\alpha \Delta r_{0}} z+\sqrt{\alpha \Delta r_{1}} z_{1}+\sqrt{\alpha \Delta r_{2}} z_{2}-\vartheta\right] s-\frac{\alpha}{2}[\beta \Delta \hat{r}-1] s^{2} .
$$

Conventions regarding averaging brackets are the same as before.

From the structure of the $1 \mathrm{RSB}$ and the 2RSB equations, a formulation of the infinite RSB scheme is fairly easily obtained. Since we have not evaluated this limit numerically, we will not reproduce the corresponding equations here. The interested reader will find the details in [16].

\section{Results}

We have solved the fixed point equations corresponding to the RS, the 1RSB and the $2 \mathrm{RSB}$ approximations for the Hopfield model with $\bar{a}=1 / 2$, as well as those corresponding to the RS and the 1RSB approximations for the generalized model at $\bar{a}=0.1$. In both cases, simulation data are available for comparison with theoretical results [7-9]. The RS approximations reproduce previously known results as they should.

As usual, the numerics simplifies considerably in the $T=0$ limit, because the innermost Gaussian averages in the saddle point equations can be performed analytically in this limit, giving simple expressions in terms of error functions. Moreover, it can be shown that, as this limit is taken, the partitioning parameters $m$ and $m_{1}, m_{2}$ of the 1RSB and the 2RSB approximations enter the theory only through the scaled combinations $D=\beta \mathrm{m}$ and $D_{1}=\beta m_{1}, D_{2}=\beta m_{2}$, which remain finite as the $\beta \rightarrow \infty$ limit is taken. In the case of the 1RSB approximations, this has already been noticed by Crisanti et al. [3].

\subsection{The standard model at $\bar{a}=1 / 2$}

In the case of the standard model, we have $A=-I=1$ and $\vartheta=0$. The full phase diagram in the RS approximation is well known [7]. In Fig. 1 we present, for later reference, an enlarged portion of it, exhibiting the boundary of the retrieval phase at low temperatures, as well as its AT-line. A noteable feature here is the reentrant behaviour signified by a 
backbending of the transition line for $T \leq T\left(\alpha_{\max }\right)=0.024$, previously discovered by Naef and Canning [4]. Moreover, the AT-line below which the RS approximation fails to be thermodynamically acceptable is seen to meet the RS phase boundary just slightly above $T\left(\alpha_{\max }\right)$, where reentrant behaviour begins. We will return to discussion of these features in Sec.5 below.

Fig. 1

Knowing that replica symmetry must be broken below the AT-line, we have analyzed the 1 RSB approximation of the model. Fig. 2 shows the order parameters $m^{1}$, $q_{1}$, and $q_{0}(\hat{q}=1)$ at $T=0.02$, for a range of $\alpha$ values that cross the AT-line at $\alpha_{\mathrm{AT}} \simeq 0.1376$. As is to be expected $q_{1}$ and $q_{0}$ become different, as $\alpha$ is increased through $\alpha_{\mathrm{AT}}$. The figure exhibits both stable and unstable solutions of the order parameters. The stable and unstable branches of these order parameters all coalesce at $\alpha_{c}^{1 \mathrm{RSB}}(T) \simeq 0.13819$, signifying the tangent-bifurcation that marks the boundary of the retrieval phase at this temperature in the $1 \mathrm{RSB}$ approximation. At zero temperature, $T=0$, we find the following critical parameters

$$
\begin{aligned}
\alpha_{c} & \simeq 0.1381864895 \\
m^{1} & \simeq 0.96677 \\
q_{0} & \simeq 0.99648 \\
C & \simeq 0.05289 \\
D & \simeq 36.78
\end{aligned}
$$

These values deviate considerably from those previously reported by Crisanti et al. [3]

$$
\begin{aligned}
\alpha_{c} & \simeq 0.144 \\
m^{1} & \simeq 0.982 \\
C & \simeq 0.111 \\
D & \simeq 0.03
\end{aligned}
$$

which they obtained through a Monte-Carlo minimization of the free energy function (24), rather than by deriving and solving the associated fixed point approximations.

Fig. 2

In view of this discrepancy, we have performed several internal consistency checks of our results. Since our expression for the free energy is the same as in [3], a possible discrepancy could arise due to errorneous expressions for the fixed point equations. However, our 1RSB fixed point system can in principle also be derived from the $2 \mathrm{RSB}$ approximation by either taking the limit $m_{2} \rightarrow 1$ or the limit $m_{1} \rightarrow n$. Both checks confirmed the expressions given in (25 29). Moreover, we have some internal consistency checks by isolating asymptotics of various integrations analytically, with essentially no 
change on the results. We have also omitted the stationarity requirement with respect to the partitioning parameter $m$, treating $D=\beta m$ as a free parameter, and computing $\alpha_{c}$ at $T=0$ as a function of $D$. The result is shown in Fig. 3. The capacity $\alpha_{c}(D)$ never increases beyond $\alpha_{c}^{1 \mathrm{RSB}}(D)_{\max } \simeq 0.1382$, approaching - as it should - the replica symmetric capacity $\alpha_{c}^{\mathrm{RS}}=0.137905$ as $D \rightarrow 0$ or $D \rightarrow \infty$, albeit in the latter case slowly. Even $\alpha_{c}^{1 \mathrm{RSB}}(D)_{\max }$ is found to be slightly smaller than $\alpha_{c}^{\mathrm{RS}}(T)_{\max } \simeq 0.1381885$. Lastly, an independent check of our results was recently obtained by Huyghebaert [12], using the bifurcation finding software package 'AUTO', and confirming our results to an accuracy of 9 significant digits.

Fig. 3

\begin{tabular}{|c|c|c|c|c|c|c|c|}
\hline & $\alpha_{c}$ & $m^{1}$ & $D$ & $D_{1}$ & $D_{2}$ & $f=u$ & $s$ \\
\hline \hline RS & 0.137905566 & 0.967417 & & & & -0.501445395 & -0.001445 \\
\hline 1RSB & 0.138186489 & 0.966777 & 36.783 & & & -0.501446051 & -0.000104 \\
\hline 2RSB & 0.138187733 & 0.966776 & & 2.406 & 38.320 & -0.501446125 & -0.000097 \\
\hline
\end{tabular}

Table 1: Retrieval boundary at $T=0$ in RS, 1RSB and 2RSB approximations for the standard model with $\bar{a}=1 / 2$. Also given are values for (free) energy $u$ and entropy $s$.

We have also considered the 2RSB approximation in the $T \rightarrow 0$ limit. Because of additional integrations that need to be performed numerically, these results are inherently less precise than those for the 1RSB scheme. They are collected in Tab. 1.

Note that the 2RSB approximation gives only a rather slight increase in the $T=0$ storage capacity, which is still within the RS reentrant phase, i.e., $\alpha_{c}^{2 \mathrm{RSB}}<\alpha_{c}^{\mathrm{RS}}(T)_{\max }$. Fig. 4 shows the Parisi function, as a function of the rescaled partitioning parameters $D_{i}=\beta m_{i}$, in the $1 \mathrm{RSB}$ and the $2 \mathrm{RSB}$ approximations, respectively.

Fig. 4

\subsection{The general model at $\bar{a}=0.1$}

For the general model ( $\bar{a}$ different from $1 / 2$ ), there is no longer a symmetry between the active and inactive neural states. In what follows we shall exclusively deal with the $\bar{a}=0.1$ case, for which simulations results are available for comparision [9]. A non-zero threshold has to be introduced, and it must be optimized in order to yield the largest retrieval region. In Fig. 5, we show the RS boundary of the retrieval region for the generalized model at $\bar{a}=0.1$, for various values of the threshold $\vartheta$. The optimal threshold, yielding the largest $T=0$ capacity is found to be $\vartheta_{\text {opt }} \simeq 1.82557$ for this system. 


\begin{tabular}{|c|c|c|c|c|c|c|}
\hline & $\alpha_{c}$ & $m^{1}$ & $C$ & $D$ & $f=u$ & $s$ \\
\hline \hline RS & 0.484151834 & 0.836979 & 0.176688 & & -0.508557635 & -0.004886 \\
\hline 1RSB & 0.495030302 & 0.828196 & 0.055155 & 3.523 & -0.509302481 & -0.000406 \\
\hline
\end{tabular}

Table 2: Retrieval boundary at $T=0$ in the RS and the 1RSB approximations for the generalized model at $\bar{a}=0.1$ and with optimal threshold $\vartheta=1.82557$.

In principle, the envelope of the retrieval regions attainable with continuously varying $\vartheta$ gives the ultimate boundary for retrieval. This would require $\vartheta$ to vary with temperature, $\vartheta=\vartheta(T)$. Note the strong reentrant behaviour in case of near optimal threshold, clearly visible in the present case without amplification.

As in the standard model, the RS solution fails to be acceptable for $T<T_{\mathrm{AT}}(\alpha)$, given by (20), and depicted in Fig. 6 for $\vartheta=\vartheta_{\text {opt }}$. Again, exactly as in the standard model, the AT-line is seen to meet the RS phase boundary just slightly above $T\left(\alpha_{\max }\right)$ below which the RS phase boundary bends back to lower values of $\alpha$. For the present case $T\left(\alpha_{\max }\right) \simeq 0.24$ at $\alpha_{\max } \simeq 0.49517$, whereas the $T=0$ capacity is

$$
\alpha_{c}^{\mathrm{RS}} \simeq 0.48415
$$

We have investigated the 1RSB approximation of this model at $T=0$, with results collected in Tab. 2.

Fig. 5,6

The $q$ values for this case are at $\hat{q}=q_{1} \simeq 0.929310$ and $q_{0} \simeq 0.891226$ compared to the replica symmetric $\hat{q}=q \simeq 0.924081$. While the relative increase in computed $T=0$ storage capacity due to $1 \mathrm{RSB}$ corrections is significantly larger than in the standard model (roughly $2 \%$ as opposed to only $0.15 \%$ in the standard model), it does still give a capacity within the reentrant phase, and far below the number $\alpha_{c}^{\text {sim }}=0.585 \pm 0.01$ reported from numerical simulations [9]. Again we have checked our results by treating $D$ as an independent parameter, not fixed by a stationarity requirement, with results qualitatively similar to the case of the standard model; see Fig. 7.

Fig. 7

Though we have not analyzed the 2RSB approximation, we expect the outcome of such an analysis to be qualitatively similar to the standard case: there will be an additional slight increase in the $T=0$ storage capacity, but it will still be smaller than $\alpha_{c}^{\mathrm{RS}}(T)_{\max }$, i.e. still be within the reentrant phase.

A final note here concerns the sharp bends in the RS phase diagrams, at temperatures above $T\left(\alpha_{\max }\right)$. They are due to the fact that the RS retrieval phase loses stability in different directions giving way to different frozen phases, depending on whether $\alpha<\alpha^{*}$ 
or $\alpha>\alpha^{*}$, where $\alpha^{*}$ denotes the loading level at which the sharp bend occurs. Since the non-retrieval phases are not so much of concern to us in the present context, we will not elaborate on this point, however. The interested reader may consult [1] and [16] on this matter.

\section{Relation with RSB in the SK-model}

In the previous section, we have seen that $1 \mathrm{RSB}$ or $2 \mathrm{RSB}$ approximations to the mean-field solution of generalized Hopfield-type models yield a slight increase of the storage capacities of these models, but this increase was found to be much smaller than probably expected from simulation results [7-9], or previously reported [3]. In all cases, the resulting storage capacity was found to be smaller than that maximally attainable in the RS approximation at finite temperature. In Sect. 4, we have also evaluated $T=0$ energies and entropies; cf. Tab. 1 and 2. The results show that the 1RSB and 2RSB approximations are still not thermodynamically acceptable at very low temperatures near the respective $\alpha_{c}$, because the $T=0$ entropies turn out to be negative, which is strictly forbidden in a system with discrete variables.

Internal energy $u$ and entropy $s$ are computed from the relations $u=\frac{\partial \beta f}{\partial \beta}$ and $s=$ $\beta u-\beta f$ in units of $k_{\mathrm{B}}$. The computations are straightforward, if perhaps tedious in details. For the $T=0$ entropy, one obtains

$$
s_{T=0}=-\frac{\alpha}{2}\left(\frac{C}{1-C}+\ln (1-C)\right),
$$

which is formally independent of the degree of approximation in the finite-step RSB scheme, provided the response parameter $C$ is defined as $C=\beta(\hat{q}-q(1))$, with $\hat{q}$ the diagonal entry in the matrix of Edwards-Anderson order parameters, and $q(1)$ the offdiagonal entry in the innermost blocks, that is $q(1)=q$ in the RS approximation, and $q(1)=q_{k}$ in the kRSB scheme, $k=1,2, \ldots$. Thus as long as $C(T=0)$ is non-zero, the $T=0$ entropy will come out negative.

The same formal independence for the zero entropy expression of the degree of approximation in the Parisi scheme is observed for the SK-model, where

$$
s \underset{T=0}{\mathrm{SK}}=-\frac{C^{2}}{4},
$$

with $C=\beta(\hat{q}-q(1))=\beta(1-q(1))$, and $q(1)=q_{k}$ in the kRSB scheme.

As a consequence, it is to be expected that no finite approximation in Parisi's approximating scheme will yield thermodynamically acceptable solutions at $T=0$ for the retrieval phases of generalized Hopfield models, just as in the case of the SK-model, where only the full hierarchical scheme of infintely many levels of RSB gives a Parisi function $q(x)$ that is sufficiently smooth on the $D=\beta x$ scale to produce a vanishing $C$ in the $T=0$ limit, and thereby a vanishing zero temperature entropy.

Further evidence for the analogy between the retrieval phases of generalized Hopfield models and the magnetic phase of the SK-model with ferromagnetic anisotropy $\left\langle J_{i j}\right\rangle=$ 
$J_{o} / N$ comes from comparing lines of constant magnetization in a RS approximation; see Fig. 8.

Fig. 8

As in the Hopfield model, reentrance is observed in the RS approximation of the SKmodel phase diagram. Moreover, in both models, the AT-line is seen to intersect the constant-magnetization lines slightly above the temperature where they begin to bend back (to larger $J_{0}$ in the SK-model, to smaller $\alpha$ in the Hopfield model). Thus, reentrant behaviour as observed in RS approximations is found to be AT unstable in both models, in a strikingly similar fashion.?

Now according to the Parisi-Toulouse hypothesis [13], one effect of RSB in the SKmodel is, roughly, to freeze the value of the magnetization as a function of temperature. That is, in the full RSB solution of the SK-model, the iso-magnetization lines in the phasediagram 7 will be verticals below the AT line. This statement is believed to be exact for the $m=0$ line, i.e., the phase boundary, and to constitute a very precise approximation otherwise [13].

By analogy, and in view of the great similarity of the analytic structure, the same is expected to hold in the case of generalized Hopfield models. As a consequence, the retrieval phase boundary - as the envelope of iso-overlap lines with non-zero $m^{\nu}$ should in the full RSB scheme turn out to be vertical (or very close to a vertical) below the point where the AT line touches the RS phase boundary.

This hypothesis is completely in accord with the results of our 1RSB and 2RSB analyses, which showed that the $T=0$ transition point $\alpha_{c}$ is shifted to slightly higher values, closer to the point where they are expected to be if the hypothesis were true, and never beyond the abscissa $\alpha^{\mathrm{RS}}(T)_{\max }$ of the reentrant point, in contrast to previously reported results [3].

\section{Summary and Discussion}

We have studied effects of RSB in generalized Hopfield-type models of attractor neural networks. We have obtained 1RSB and 2RSB corrections to RS results, which are much smaller than expected from simulation results [7-9] or previously reported for the standard model at $\bar{a}=1 / 2[3]$. In all cases the 1RSB and 2RSB storage capacities obtained were found to be smaller than those attainable in the RS approximation at finite temperature. Our results were found to be consistent with what is to be expected if the Parisi-Toulouse hypothesis about the nature of the Parisi function $q(x)[11,13]$ would hold for Hopfieldtype models in the same manner as it does in the SK spin glass model. On the basis of this hypothesis, we conjecture that the reentrance phenomenon observed in RS analyses of Hopfield-type models would simply disappear in a full hierarchical Parisi RSB solution

\footnotetext{
${ }^{1}$ There is a mapping of the replica symmetric Hopfield model onto the replica symmetric SK-model; for details see [16].
} 
of these models.

This state of affairs raises the question of how to reconcile discrepancies between theory and numerical experiments in these systems. Two possible explanations come to mind, and both are, we think, worth checking.

One possibility is that $T=0$-Monte Carlo Dynamics gets trapped in energy valleys which are surrounded by nonextensive energy barriers, $\Delta E \sim N^{\nu}$ with $0 \leq \nu<1$. The existence of such nonextensive energy barriers between thermodynamically unstable retrieval states and the spin glass phase might well invalidate conventional (exponential) finite-size-scaling expressions for first order phase transitions, on which the analyses of simulation data [7,9] were based. Here we should, however, remark that Müller [17] — knowing of our results - has recently performed Monte Carlo simulations which would confirm our values for $\alpha_{c}$ on the basis of a conventional finite-size-scaling analysis of his data.

A second possibility concerns the existence of dynamically frozen phases not detectable in equilibrium treatments. Discrepancies analogous to those between our results and those of simulations have, indeed, recently been observed in the case of the binary perceptron [18], the $p$-spin interaction spin glass [19], and in the case of fluctuating manifolds in random media [20], where dynamically frozen phases were observed in regions of parameter space in which static approaches yielded ergodic phases.

Acknowledgements This work has been part of the PhD thesis of HS. Numerous fruitful discussions with $\mathrm{H}$. Horner are gratefully and with pleasure acknowledged. RK thanks the Laboratoire de Physique Théorique of ENS for the hospitality extended to him while parts of this paper were being written.

\section{References}

[1] H. Horner, Z. Phys. B 75, 133 (1989)

[2] C.J. Perez-Vicente and D.J. Amit, J. Phys. A 22, 559 (1989)

[3] A. Crisanti, D.J. Amit, and H. Gutfreund, Europhys. Lett. 2, 337 (1986)

[4] J.P. Naef and A. Canning, J. Phys. 2, 247 (1992)

[5] J.J. Hopfield, Proc. Natl. Acad. Sci. USA 79, 2554 (1982)

[6] D. Sherrington and S. Kirkpatrick, Phys. Rev. Lett. 35, 1792 (1975); S. Kirkpatrick and D. Sherrington, Phys. Rev. B 17, 4384 (1978); see also M. Mézard, G. Parisi, and M. A. Virasoro, Spin Glass Theory and Beyond, (World Scientific, Singapore, 1987)

[7] D.J. Amit, H. Gutfreund, and H. Sompolinsky, Ann. Phys. (N.Y.) 173, 30 (1987) 
[8] G.A. Kohring, J. Stat. Phys. 59, 1077 (1990)

[9] B.M. Forrest und A. Loettgers, Z. Phys. B 88, 309 (1992)

[10] G. Parisi, J. Phys. A13, L115 (1980)

[11] G. Parisi, J. Phys. A13, 1101 (1980)

[12] J. Huyghebaert, private Communication; see also D. Bollé and J. Huyghebaert, Mixture states and the storage of biased patterns in the Hopfield model: a replica-symmetry breaking solution, preprint (Leuven, 1993) KUL-TF$93 / 46$

[13] G. Parisi and G. Toulouse, J. Phys. (Paris) Lett. 41, L361 (1980); J. Vannimenus, G. Toulouse, and G. Parisi, J. Phys. (Paris) 42, 565 (1981)

[14] E. Gardner, Europhys. Lett. 4, 481 (1987); —, J. Phys. A 21, 257-270 (1988)

[15] J.R.L. de Almeida and D.J. Thouless, J. Phys. A11, 983 (1978)

[16] H. Steffan, Replikasymmetriebrechung in Attraktor-Neuronalen-Netzen, PhD-Thesis, Heidelberg (1993), unpublished

[17] K.R. Müller, private communication (1993)

[18] H. Horner, Z. Phys. B 86291 (1992)

[19] A. Crisanti and H.J. Sommers, Z. Phys. B 87341 (1992); A. Crisanti, H. Horner, and H.J. Sommers, Z. Phys. B 92257 (1993)

[20] M. Mézard and G. Parisi, J. Phys. A23, L1229 (1990); —, J. Phys. I France 1809 (1991); H. Kinzelbach and H. Horner, J. Phys. I France 31329 (1993); — ibid. 31901 (1993) 


\section{A The 1RSB Approximation}

In this appendix, we present the main ideas that go into the evaluation of the replica free energy (8) in the 1RSB approximation, and into the derivation of the corresponding $1 \mathrm{RSB}$ version of the fixed point equations (13). The 1RSB approximation is based on the ansatz (23) for the matrix of Edwards-Anderson order parameters, in which $m$ is a partitioning parameter for the set of $n$ replica that is to be determined from a stationarity condition on $f$. Clearly, the structure of $\mathbf{q}$ is inherited by $\mathbf{1}-\beta \mathbf{q}$,

$$
\mathbf{1}-\beta \mathbf{q}=\left(1-\beta\left(\hat{q}-q_{1}\right)\right) \mathbf{1}_{n}-\beta\left(q_{1}-q_{0}\right) \mathbf{1}_{\frac{n}{m}} \otimes \mathbf{e}_{m} \mathbf{e}_{m}^{\mathrm{T}}-\beta q_{0} \mathbf{e}_{n} \mathbf{e}_{n}^{\mathrm{T}},
$$

as well as by $\mathbf{r}$ in virtue of (14). Using this structure, we have to evaluate the various terms appearing in the free energy (8).

(i) In order to evaluate $\ln \operatorname{det}(\mathbf{1}-\beta \mathbf{q})$, we diagonalize (47). This is accomplished by noting that $\mathbf{1}_{n}=\mathbf{1}_{\frac{n}{m}} \otimes \mathbf{1}_{m}$, and $\mathbf{e}_{n} \mathbf{e}_{n}^{\mathrm{T}}=\mathbf{e}_{\frac{n}{m}} \mathbf{e}_{\frac{n}{m}}^{\mathrm{T}} \otimes \mathbf{e}_{m} \mathbf{e}_{m}^{\mathrm{T}}$, and that matrices with tensor product structure can be diagonalized separately in each tensor product component. Matrices of the form $\mathbf{e}_{k} \mathbf{e}_{k}^{\mathrm{T}}$ have one eigenvalue $k$ and a $(k-1)$-fold eigenvalue zero. Since they trivially commute with the corresponding unit matrices $\mathbf{1}_{k}$, the full spectrum of (47) is readily obtained to yield

$$
\begin{aligned}
\operatorname{det}(\mathbf{1}-\beta \mathbf{q})= & \left(1-\beta\left(\hat{q}-q_{1}\right)-\beta m\left(q_{1}-q_{0}\right)-n \beta q_{0}\right) \\
& \left(1-\beta\left(\hat{q}-q_{1}\right)-\beta m\left(q_{1}-q_{0}\right)\right)^{\frac{n}{m}-1} \\
& \left(1-\beta\left(\hat{q}-q_{1}\right)\right)^{n-\frac{n}{m}}
\end{aligned}
$$

(ii) Next, the term $\sum_{a \leq b} r_{a b} q_{a b}$ appearing in (8) is evaluated by endowing $\mathbf{r}$ with the same block structure as $\mathbf{q}$, and by parametrizing it analogously. This gives

$$
\sum_{a \leq b} r_{a b} q_{a b}=\frac{1}{2}\left(n \hat{r} \hat{q}+\sum_{a, b} r_{a b} q_{a b}\right)=\frac{n}{2}\left[2 \hat{r} \hat{q}+(m-1) r_{1} q_{1}+(n-m) r_{0} q_{0}\right]
$$

(iii) In view of (14), to express the elements of $\mathbf{r}$ in terms of those of $\mathbf{q}$, we have to invert $\mathbf{1}-\beta \mathbf{q}$. This is done by noting that the matrices $\mathbf{1}_{k}$ and $\mathbf{e}_{k} \mathbf{e}_{k}^{\mathrm{T}}$ form a closed algebra, since $\mathbf{e}_{k} \mathbf{e}_{k}^{\mathrm{T}} \mathbf{e}_{k} \mathbf{e}_{k}^{\mathrm{T}}=k \mathbf{e}_{k} \mathbf{e}_{k}^{\mathrm{T}}$. Thus the inverse of $\mathbf{1}-\beta \mathbf{q}$ must be of the same structure as $\mathbf{1}-\beta \mathbf{q}$ itself, albeit with different coefficients in front of the three tensor product matrices appearing in (47). These coefficients are computed from the condition that the product of $\mathbf{1}-\beta \mathbf{q}$ and its inverse should give a unit matrix. This yields (28), with the $Q_{q_{i}}$ defined by (27).

(iv) Finally, to evaluate the single-site replica partition function corresponding to the Hamiltonian (10), we have to decouple the replicated spins coupled through the term $\sum_{a \leq b} r_{a b} s^{a} s^{b}$. With $\mathbf{r}$ of 1RSB form analogous to (23), we get

$$
\sum_{a \leq b} r_{a b} s^{a} s^{b}=\frac{1}{2}\left[\left(2 \hat{r}-r_{1}\right) \sum_{a=1}^{n}\left(s^{a}\right)^{2}+\left(r_{1}-r_{0}\right) \sum_{k=1}^{n / m}\left(\sum_{a=1}^{m} s^{(k-1) m+a}\right)^{2}+r_{0}\left(\sum_{a=1}^{n} s^{a}\right)^{2}\right] .
$$


Gaussian linearization of the last term in (50), using a Gaussian variable $z$, will produce $n / m$ identical uncoupled blocks of size $m$ in the evaluation of (9). Within these blocks there is still a coupling between spins due to the next-to-last term in (50). These are decoupled using a Gaussian $z_{1}$. This then yields

$$
\langle\ln \hat{Z}\rangle_{\xi^{\nu}}=n\left\langle\ln \left\langle\tilde{Z}^{m}\right\rangle_{z_{1}}^{\frac{1}{m}}\right\rangle_{z, \xi^{\nu}}
$$

in (8), as $n \rightarrow 0$, with $\tilde{Z}$ the partition function corresponding to the on-site Hamiltonian (29). Similar linearization techniques are readily seen to produce the $1 \mathrm{RSB}$ fixed point equations (25) from (13), as the $n \rightarrow 0$-limit is taken.

Collecting all items so far computed, we obtain the 1RSB expression (24). Stationarity of this expression with respect to the partitioning parameter $m$ requires (26) to hold, which completes our derivation of the 1RSB approximation.

\section{B The 2RSB Approximation}

In the present appendix, we sketch the evaluation of (8) in the 2RSB approximation, and the derivation of the corresponding 2RSB version of the fixed point equations (13). The following outline completely parallels that of Appendix A, and we will not repeat the arguments in detail.

The 2RSB approximation is based on the ansatz (30) for the matrix of EdwardsAnderson order parameters, in which $m_{1}$ and $m_{2}$ are partitioning parameters which are to be determined from a stationarity condition on $f$. Again, the structure of $\mathbf{q}$ is inherited by $1-\beta \mathbf{q}$,

$$
\begin{aligned}
\mathbf{1}-\beta \mathbf{q}= & \left(1-\beta\left(\hat{q}-q_{2}\right)\right) \mathbf{1}_{n}-\beta\left(q_{2}-q_{1}\right) \mathbf{1}_{\frac{n}{m_{2}}} \otimes \mathbf{e}_{m_{2}} \mathbf{e}_{m_{2}}^{\mathrm{T}} \\
& -\beta\left(q_{1}-q_{0}\right) \mathbf{1}_{\frac{n}{m_{1}}} \otimes \mathbf{e}_{m_{1}} \mathbf{e}_{m_{1}}^{\mathrm{T}}-\beta q_{0} \mathbf{e}_{n} \mathbf{e}_{n}^{\mathrm{T}} .
\end{aligned}
$$

as well as by $\mathbf{r}$ in virtue of (14).

(i) In order to evaluate $\ln \operatorname{det}(\mathbf{1}-\beta \mathbf{q})$, we diagonalize (52) along the lines outlined in Appendix A. This yields

$$
\begin{aligned}
\operatorname{det}(\mathbf{1}-\beta \mathbf{q})= & \left(1-\beta\left(\hat{q}-q_{2}\right)-\beta m_{2}\left(q_{2}-q_{1}\right)-\beta m_{1}\left(q_{1}-q_{0}\right)-\beta n q_{0}\right) \\
& \left.\left(1-\beta\left(\hat{q}-q_{2}\right)-\beta m_{2}\left(q_{2}-q_{1}\right)-\beta m_{1}\left(q_{1}-q_{0}\right)\right)\right)^{\frac{n}{m_{1}}-1} \\
& \left.\left(1-\beta\left(\hat{q}-q_{2}\right)-\beta m_{2}\left(q_{2}-q_{1}\right)\right)\right)^{\frac{n}{m_{2}}-\frac{n}{m_{1}}} \\
& \left(1-\beta\left(\hat{q}-q_{1}\right)\right)^{n-\frac{n}{m_{2}}} .
\end{aligned}
$$

(ii) The term $\sum_{a \leq b} r_{a b} q_{a b}$ appearing in (8) is evaluated by endowing $\mathbf{r}$ with the same block structure as $\mathbf{q}$, and by parametrizing it analogously. This gives

$$
\sum_{a \leq b} r_{a b} q_{a b}=\frac{n}{2}\left[2 \hat{r} \hat{q}+\left(m_{2}-1\right) r_{2} q_{2}+\left(m_{1}-m_{2}\right) r_{1} q_{1}+\left(n-m_{1}\right) r_{0} q_{0}\right]
$$


(iii) To express the elements of $\mathbf{r}$ in terms of those of $\mathbf{q}$, we have to invert $\mathbf{1}-\beta \mathbf{q}$. This is done as outlined in Appendix A, and yields (39), with the $Q_{q_{i}}$ defined by (40).

(iv) Next, to evaluate the single-site replica partition function corresponding to the Hamiltonian (10), we have to decouple the replicated spins coupled through the term $\sum_{a \leq b} r_{a b} s^{a} s^{b}$. With $\mathbf{r}$ of 2 RSB form analogous to (30), we get

$$
\begin{aligned}
\sum_{a \leq b} r_{a b} s^{a} s^{b}=\frac{1}{2} & {\left[\left(2 \hat{r}-r_{2}\right) \sum_{a=1}^{n}\left(s^{a}\right)^{2}+\left(r_{2}-r_{1}\right) \sum_{k=1}^{n / m_{2}}\left(\sum_{a=1}^{m_{2}} s^{(k-1) m_{2}+a}\right)^{2}\right.} \\
& \left.+\left(r_{1}-r_{0}\right) \sum_{k=1}^{n / m_{1}}\left(\sum_{a=1}^{m_{1}} s^{(k-1) m_{1}+a}\right)^{2}+r_{0}\left(\sum_{a=1}^{n} s^{a}\right)^{2}\right]
\end{aligned}
$$

This structure suggests an iterative Gaussian linearization scheme as in the case of the 1RSB approximation dicussed in the previous appendix. A Gaussian $z$ is introduced to decouple spins in different blocks of size $m_{1}$, and creates $n / m_{1}$ identical independent, i.e., uncoupled blocks of this size. Within a block of size $m_{1}$, there are $m_{1} / m_{2}$ identical blocks of size $m_{2}$, which are decoupled through a Gaussian $z_{1}$. Finally the $m_{2}$ spins within each of these smaller blocks are decoupled using a Gaussian $z_{2}$. This results in

$$
\langle\ln \hat{Z}\rangle_{\xi^{\nu}}=n\left\langle\ln \left\langle\left\langle\tilde{Z}^{m_{2}}\right\rangle_{z_{2}}^{\frac{m_{1}}{m_{2}}}\right\rangle_{z_{1}}^{\frac{1}{m_{1}}}\right\rangle_{z, \xi^{\nu}}
$$

in (8), as $n \rightarrow 0$. Here $\tilde{Z}$ is the partition function corresponding to the on-site Hamiltonian (41). Again, similar linearization techniques are seen to produce the 2RSB fixed point equations (36) from (13), as the $n \rightarrow 0$-limit is taken.

Collecting all items, we obtain the 2RSB expression (31). Stationarity of this expression with respect to the partitioning parameters $m_{1}$ and $m_{2}$ requires (37) and (38) to hold, which completes our derivation of the 2RSB approximation. 